\title{
Correction to: New Properties on Normalized Null Hypersurfaces
}

\author{
Cyriaque Atindogbe, Manuel Gutiérrez(B) \\ and Raymond Hounnonkpe
}

\section{Correction to: Mediterr. J. Math. (2018) 15:166 https://doi.org/10.1007/s00009-018-1210-0}

There is a mistake in the last subsection (5.3 Totally Geodesic Null Hypersurfaces in Robertson-Walker Spaces) of the original paper. In fact, in the proof of Theorem 5.16 we used the formula $\widetilde{K}^{S}(X, Y)=\frac{c-\left(f^{\prime}\right)^{2}}{f^{2}}$ for every $X, Y \in \mathfrak{X}(S)$ where $S$ is a leaf of the screen distribution, whereas the correct one is $\widetilde{K}^{S}(X, Y)=\frac{c+\left(f^{\prime}\right)^{2}}{f^{2}}$. It follows that all the arguments used in the proof of Theorem 5.16 and Corollary 5.17 are not valid and cannot be fixed without rewriting the whole subsection.

This is what we want to do in this erratum. Following the aims of the subsection, we can study the implications of the use of the rigging technique in the properties of totally geodesic null hypersurfaces. Notice that the new results are applicable to the more general setting of Generalized RobertsonWalker spaces. The new subsection should be:

\subsection{Totally Geodesic Null Hypersurfaces in Generalized Robertson-Walker Spaces}

Totally geodesic null hypersurfaces are very used in general relativity as they represent horizons of various sorts (non expanding, isolated, Killing, etc.). We investigate here about the topological properties of closed (in the topological sense) totally geodesic null hypersurfaces and prove that they are foliated by closed marginally trapped submanifolds in certain Generalized RobertsonWalker spaces.

The original article can be found online at https://doi.org/10.1007/s00009-018-1210-0. 
Theorem 0.1. Let $\bar{M}=\mathbb{R} \times_{f} F^{n+1}$ be a Generalized Robertson-Walker space with complete Riemannian factor. Suppose $f$ is strictly monotone and there exists a positive constant $k$ such that $K^{F}+\left(f^{\prime}\right)^{2}>k$ (being $K^{F}$ the sectional curvature of $F)$. Then any topologically closed embedded totally geodesic null hypersurface $M$ is diffeomorphic to $\mathbb{R} \times S$, where $S$ is a compact manifold with finite fundamental group and $\{t\} \times S$ are marginally trapped submanifold.

Proof. Suppose there exists a topologically closed embedded totally geodesic null hypersurface $M$ in $\bar{M}$. Consider the normalizing rigging $\zeta=\sqrt{2} \frac{\partial}{\partial t}$ for $M$, so its associated rigged vector field $\xi$ is a null past vector field.

A leaf $S$ of $\mathscr{S}(\zeta)$ is the intersection of $M$ with a fiber of the time function $t$, hence it is a closed submanifold contained in some slice $\left\{t_{0}\right\} \times F^{n+1}$. Let us call $g$ the Riemannian metric on $F^{n+1}$. Since the Lorentzian metric on $\bar{M}$ is given by $\bar{g}=-d t^{2}+f^{2} g$, we get that $\widetilde{g}_{\left.\right|_{S}}=f^{2}\left(t_{0}\right) g$ so that $\left(S, \widehat{g}=\frac{1}{f^{2}\left(t_{0}\right)} \widetilde{g}_{\left.\right|_{S}}\right)$ is isometric to a hypersurface of $F^{n+1}$.

The following relation holds, [2]

$$
\begin{aligned}
& \bar{K}(X, Y)-\widetilde{K}^{S}(X, Y) \\
& \quad=-C(X, X) B(Y, Y)-B(X, X) C(Y, Y)+2 C(X, Y) B(X, Y)
\end{aligned}
$$

$\forall X, Y \in \mathscr{S}(\zeta)$, where $\bar{K}(X, Y)$ is the sectional curvature in $(\bar{M}, \bar{g})$ and $\widetilde{K}^{S}(X, Y)$ the induced sectional curvature from $(M, \widetilde{g})$. Since the null hypersurface $M$ is totally geodesic, $B$ vanishes identically. Hence

$$
\widetilde{K}^{S}(X, Y)=\bar{K}(X, Y)=\frac{K^{F}+\left(f^{\prime}\right)^{2}}{f^{2}}
$$

$\forall X, Y \in \mathscr{S}(\zeta)$. Finally since $\widehat{g}=\frac{1}{f^{2}\left(t_{0}\right)} \widetilde{g}_{\left.\right|_{S}}$, the sectional curvature of the hypersurface $(S, \widehat{g})$ is

$$
\widehat{K}=K^{F}+\left(f^{\prime}\right)^{2}\left(t_{0}\right)>k>0 .
$$

Bonnet-Myers theorem implies $S$ is a compact manifold with finite fundamental group. Using the original paper, $(M, \widetilde{g})$ is complete, so being unitary the rigged vector field $\xi$ is complete. An argument as in $[2$, Theorem 5.3 and Remark 5.4] shows that $M$ is diffeomorphic to $\mathbb{R} \times S$.

The second fundamental form $I I$ of $S$ in $(\bar{M}, \bar{g})$ is given by

$$
I I(X, Y)=C(X, Y) \xi+B(X, Y) N
$$

$\forall X, Y \in \mathscr{S}(\zeta)$ but $B=0$, hence

$$
I I(X, X)=C(X, X) \xi .
$$

Since $\zeta$ is orthogonally conformal, $\left(L_{\zeta} g\right)(X, X)=\rho g(X, X)$ where the conformal factor is $\rho=2 \sqrt{2} \frac{f^{\prime}}{f}$. From [2, Corollary 3.6] we have

$$
C(X, X)=-\frac{\rho}{2}
$$

$\forall X$ unitary in $\mathscr{S}(\zeta)$, then

$$
I I(X, X)=-\frac{\rho}{2} \xi .
$$


Taking the trace of $I I$, we get that the mean curvature vector field of $S$ is given by $H^{S}=-\frac{\rho}{2} \xi$. Since $f$ is strictly monotone, $\rho \neq 0$, hence $H^{S}$ is a null vector field and so $S$ is a closed marginally trapped submanifold in $(\bar{M}, \bar{g})$.

In the special case of $\operatorname{dim} \bar{M}=4$, if $F$ is orientable, $S$ is diffeomorphic to the sphere $\mathbb{S}^{2}$.

In [1, Theorem IV.1.], Galloway shows that if a Lorentzian manifold is null complete and satisfies the null convergence condition then any null line is contained in a smooth (topologically) closed embedded achronal totally geodesic null hypersurface. So the following holds.

Corollary 0.2. Let $\bar{M}=\mathbb{R} \times_{f} F^{n+1}$ be a Generalized Robertson-Walker space with complete Riemannian factor. Suppose $f$ is strictly monotone and there exists a positive constant $k$ such that $K^{F}+\left(f^{\prime}\right)^{2}>k$. If $(\bar{M}, \bar{g})$ is null complete and satisfies the null convergence condition, then one of the following holds.

1. $(\bar{M}, \bar{g})$ contains no null line.

2. $(\bar{M}, \bar{g})$ admits a closed marginally trapped codimension two submanifold.

Proof. Suppose point 1 does not hold, then from the above mentioned Galloway's theorem, there exists a topologically closed totally geodesic null hypersurface containing the null line. From Theorem 0.1, there exists a closed marginally trapped submanifold in $(\bar{M}, \bar{g})$.

\section{Acknowledgements}

The second and third authors have been partially supported by an FEDERMTM2016- 78647-P grant. The third author has been partially supported by a CEA-SMA grant.

\section{References}

[1] Galloway, G.J.: Maximum principles for null hypersurfaces and null splitting theorems. Ann. Henri Poincaré 1, 543-567 (2000)

[2] Gutiérrez, M., Olea, B.: Induced Riemannian structures on null hypersurfaces. Math. Nachr. 289, 1219-1236 (2016)

Cyriaque Atindogbe and Raymond Hounnonkpe

Institut de Mathématiques et de Sciences Physiques (IMSP)

Université d'Abomey-Calavi

Abomey-Calavi

Bénin

e-mail: atincyr@imsp-uac.org

Raymond Hounnonkpe

e-mail: rhounnonkpe@ymail.com 
Manuel Gutiérrez

Universidad de Málaga

Málaga

Spain

e-mail: m_gutierrez@uma.es 